\title{
Electromagnetic and Scalar Pion form factor in the Kroll-Lee-Zumino model
}

\author{
C. A. Dominguez ${ }^{\mathrm{a}, \mathrm{b}}$, J. I. Jottar ${ }^{\mathrm{c}, \mathrm{d}}$, M. Loewe ${ }^{\mathrm{c}}$, B. Willers ${ }^{\mathrm{a}}$ \\ ${ }^{a}$ Centre for Theoretical Physics and Astrophysics, University of Cape Town \\ Rondebosch 7700, South Africa \\ ${ }^{\mathrm{b}}$ Department of Physics, Stellenbosch University \\ Stellenbosch 7600, South Africa \\ 'Pontificia Universidad Católica de Chile, \\ Casilla 306, Santiago 22, Chile \\ ${ }^{\mathrm{d}}$ Department of Physics, University of Illinois, \\ Urbana-Champaign, IL, 61801 3080, USA
}

\begin{abstract}
The renormalizable Abelian quantum field theory model of Kroll, Lee, and Zumino is used at the one loop level to compute vertex corrections to the tree-level, Vector Meson Dominance (VMD) electromagnetic pion form factor. These corrections, together with the one-loop vacuum polarization contribution, imply a resulting electromagnetic pion form factor in excellent agreement with data in the whole range of accessible momentum transfers in the space-like region. The time-like form factor, which reproduces the Gounaris-Sakurai formula at and near the rho-meson peak, is unaffected by the vertex correction at order $\mathcal{O}\left(g^{2}\right)$. The KLZ model is also used to compute the scalar radius of the pion at the one loop level, finding $\left\langle r_{\pi}^{2}\right\rangle_{S}=0.40 \mathrm{fm}^{2}$. This value implies for the low energy constant of chiral perturbation theory $\bar{l}_{4}=3.4$.
\end{abstract}

PACS numbers:

Keywords: 
The renormalizable Abelian quantum field theory of charged pions, and massive neutral vector mesons, proposed by Kroll, Lee, and Zumino (KLZ) [1], provides a rigorous theoretical justification for the Vector Meson Dominance (VMD) ansatz [2]. Since the neutral vector mesons are coupled only to conserved currents the model is renormalizable [1],[3]. Gale and Kapusta [4] computed in this model the rho-meson self energy to one-loop order. When this result is used in the VMD expression for the electromagnetic pion form factor, the Gounaris-Sakurai formula [5]-[6] in the time-like region is found at and near the rho-meson pole. This is quite intriguing. That an empirical fit formula such as this should follow from the KLZ Lagrangian may be hinting at additional unexpected properties of this model. Here we compute the vertex diagram, i.e. the one loop correction to the strong coupling constant in the framework of the KLZ model. This correction is of the same order in the coupling as the one loop vacuum polarization. After going through regularization and renormalization, and in conjunction with the VMD expression for the electromagnetic pion form factor, this vertex correction, together with the vacuum polarization contribution, leads to an excellent agreement between theory and data in the spacelike region. The parameter free result (masses and couplings are known from experiment) represents a substantial improvement over naive (tree-level) VMD. The resulting chi-squared per degree of freedom is close to unity, while the one from tree-level VMD is about five times bigger. Time-like region predictions are unaffected by the vertex correction. In fact, the combination of vacuum polarization and vertex corrections in this region turns out to be of higher order in the coupling. Since the KLZ model involves a strong coupling, the perturbative expansion could be questioned, and the next-to-leading (one-loop) contributions need not be smaller than the leading term. However, this is not the case in the KLZ model. In fact, the relatively small $\rho \pi \pi$ coupling $\left(g_{\rho \pi \pi} \simeq 5\right)$ is accompanied by the large loop suppression factor $1 /(4 \pi)^{2}$, so that the one-loop contributions remain reasonable corrections to the leading order tree-level term. At higher orders, we expect higher powers of this suppression factor from loop integrations. A next-to-next-to leading order calculation is beyond the scope of this work.

The KLZ lagrangian is given by 


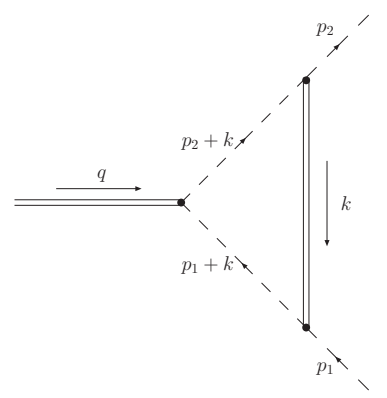

FIG. 1: Vertex function kinematics

$$
\begin{array}{r}
L_{K L Z}=\partial_{\mu} \phi \partial^{\mu} \phi^{*}-\frac{1}{4} m_{\pi}^{2} \phi \phi^{*}-\frac{1}{4} \rho_{\mu \nu} \rho^{\mu \nu} \\
+\frac{1}{2} m_{\rho}^{2} \rho_{\mu} \rho^{\mu}+g_{\rho \pi \pi} \rho_{\mu} J_{\pi}^{\mu},
\end{array}
$$

where $\rho_{\mu}$ is a vector field describing the $\rho^{0}$ meson $\left(\partial_{\mu} \rho^{\mu}=0\right), \phi$ is a complex pseudo-scalar field describing the $\pi^{ \pm}$mesons, $\rho_{\mu \nu}$ is the usual field strength tensor, and $J_{\pi}^{\mu}$ is the $\pi^{ \pm}$ current, i.e.

$$
\begin{gathered}
\rho_{\mu \nu}=\partial_{\mu} \rho_{\nu}-\partial_{\nu} \rho_{\mu}, \\
J_{\pi}^{\mu}=i\left(\phi^{*} \partial^{\mu} \phi-\left(\partial^{\mu} \phi^{*}\right) \phi\right) .
\end{gathered}
$$

It is important to remark that the KLZ lagrangian includes also a coupling of the form $g_{\rho \pi \pi}^{2} \rho_{\mu} \rho^{\mu} \phi \phi^{*}$ which we omitted from Eq.1, since it is not relevant for our calculation.

In Fig. 1 we show the vertex function kinematics. The double line denotes the $\rho$-propagator and the dashed lines the pion propagators. The technical details associated to the evaluation of this diagram can be found in the original reference [7]. Here we will only present the main results. In the Feynman gauge and using dimensional regularizarion, [3], [8] our vertex function can be written as

$$
\begin{array}{r}
\widetilde{\Gamma}_{\rho \pi \pi}^{(1) \mu}\left(p_{1}, p_{2}, q^{2}\right)=\Gamma_{\rho \pi \pi}^{(0) \mu}\left(p_{1}, p_{2}\right)\left[\widetilde{G}\left(q^{2}\right)+\right. \\
\left.A\left(\frac{2}{\varepsilon}-\frac{1}{2}-\gamma+\ln (4 \pi)\right)\right]+\mathcal{O}(\varepsilon)
\end{array}
$$

where the tree-level contribution is given by

$$
\Gamma_{\rho \pi \pi}^{(0) \mu}\left(p_{1}, p_{2}\right)=i g_{\rho \pi \pi} \mu^{\left(2-\frac{d}{2}\right)}\left(p_{1}+p_{2}\right)^{\mu}
$$


and

$$
\begin{gathered}
\widetilde{G}\left(q^{2}\right)=-2 \frac{g_{\rho \pi \pi}^{2}}{(4 \pi)^{2}} \int_{0}^{1} d x_{1} \int_{0}^{1-x_{1}} d x_{2}\left\{\left(2-3 x_{1}\right)\right. \\
\ln \left(\frac{\Delta\left(q^{2}\right)}{\mu^{2}}\right)+\left(\frac{1-2 x_{1}}{2 \Delta\left(q^{2}\right)}\right)\left[m_{\pi}^{2}\left(x_{1}+x_{2}-2\right)^{2}\right. \\
\left.\left.-q^{2}\left(x_{1} x_{2}-x_{1}-x_{2}+2\right)\right]\right\}
\end{gathered}
$$

The factor $A$ is a certain integral over $x_{1}$ and $x_{2}$ but it does not depend on $q^{2}$. So, this constant will be canceled during the renormalization procedure. In the previous equations $\mu$ is the scale mass factor associated to dimensional regularization and

$$
\Delta\left(q^{2}\right)=m_{\pi}^{2}\left(x_{1}+x_{2}\right)^{2}+m_{\rho}^{2}\left(1-x_{1}-x_{2}\right)-x_{1} x_{2} q^{2}
$$

It is convenient to choose $q^{2}=0$ as the renormalization point for the vertex function, because we can make use of the well known normalization of the pion form factor $F_{\pi}(0)=1$, together with

$$
\Gamma_{\rho \pi \pi}^{(1) \mu}\left(p_{1}, p_{2}, q^{2}=0\right)=\Gamma_{\rho \pi \pi}^{(0)}\left(p_{1}, p_{2}\right)
$$

The electromagnetic pion form factor in VMD at tree level is given by

$$
\left.F_{\pi}\left(q^{2}\right)\right|_{\mathrm{VMD}}=\frac{g_{\rho \pi \pi}}{f_{\rho}} \frac{M_{\rho}^{2}}{M_{\rho}^{2}-q^{2}} .
$$

The renormalized electromagnetic pion form factor associated to the substracted one-loop vertex corrections at $q^{2}=0$, at order $\mathcal{O}\left(g_{\rho \pi \pi}^{2}\right)$, can be written as

$$
\begin{aligned}
& \left.F_{\pi}\left(q^{2}\right)\right|_{\text {vertex }}=\left.F_{\pi}\left(q^{2}\right)\right|_{\mathrm{VMD}} \\
& \times\left[1+\widetilde{G}\left(q^{2}\right)-\widetilde{G}(0)\right],
\end{aligned}
$$

where $f_{\rho}=4.97 \pm 0.07[9]$, and from universality and $F_{\pi}(0)=1$ it follows that $g_{\rho \pi \pi}(0)=f_{\rho}$. Hence, the one-loop vertex correction generates an additional momentum dependence in the form factor.

Two seagull-type corrections of the same order in $\mathcal{O}\left(g_{\rho \pi \pi}^{2}\right)$ in principle should be included. However these diagrams do not depend on $q^{2}$ and they cancel when substracting at $q^{2}=0$. Another contribution to the pion form factor comes from the vacuum polarization corrections 
to the $\rho$-propagator. This calculation has been done in [4]. See [7] for details. Adding the vacuum polarization to the vertex contribution gives the complete correction to the VMD electromagnetic pion form factor at order $\mathcal{O}\left(g_{\rho \pi \pi}^{2}\right)$. We emphasize that the result does not contain free parameters, since the masses and the coupling are known from experiment.

$$
\begin{aligned}
& F_{\pi}\left(q^{2}\right)=\frac{M_{\rho}^{2}+\left.\Pi(0)\right|_{\mathrm{vac}}}{M_{\rho}^{2}-q^{2}+\left.\Pi\left(q^{2}\right)\right|_{\mathrm{vac}}} \\
& +\frac{M_{\rho}^{2}}{M_{\rho}^{2}-q^{2}}\left[\widetilde{G}\left(q^{2}\right)-\widetilde{G}(0)\right]
\end{aligned}
$$

where

$$
\begin{aligned}
& \left.\Pi\left(q^{2}\right)\right|_{\operatorname{vac}}=\frac{1}{3} \frac{g_{\rho \pi \pi}^{2}}{(4 \pi)^{2}} q^{2}\left(1-4 \frac{\mu_{\pi}^{2}}{q^{2}}\right)^{3 / 2} \\
& \times\left[\ln \left|\frac{\sqrt{\left(1-4 \mu_{\pi}^{2} / q^{2}\right)}+1}{\sqrt{\left(1-4 \mu_{\pi}^{2} / q^{2}\right)}-1}\right|\right. \\
& \left.-i \pi \theta\left(q^{2}-4 \mu_{\pi}^{2}\right)\right]+A q^{2}+B
\end{aligned}
$$

and where the constants $A$ and $B$ are given by

$$
\begin{gathered}
A=-\frac{1}{3} \frac{g_{\rho \pi \pi}^{2}}{(4 \pi)^{2}}\left[8 \frac{\mu_{\pi}^{2}}{M_{\rho}^{2}}+\left(1-4 \frac{\mu_{\pi}^{2}}{M_{\rho}^{2}}\right)^{3 / 2}\right. \\
\left.\times \ln \left|\frac{\sqrt{\left(1-4 \mu_{\pi}^{2} / M_{\rho}^{2}\right)}+1}{\sqrt{\left(1-4 \mu_{\pi}^{2} / M_{\rho}^{2}\right)}-1}\right|\right], \\
B=\left.\Pi(0)\right|_{\operatorname{vac}}=\frac{8}{3} \frac{g_{\rho \pi \pi}^{2}}{(4 \pi)^{2}} \mu_{\pi}^{2} .
\end{gathered}
$$

It is important to remark that the vacuum polarization correction is not included in the second term of Eq.11 as it would make this term of $\mathcal{O}\left(g^{4}\right)$. Hence, the vertex correction does not affect the form factor in the time-like region, where it becomes the Gounaris-Sakurai formula near the rho-meson peak. In fact, from the definition of the hadronic width [10]: $\Gamma_{\rho}=-\left(1 / M_{\rho}\right) \operatorname{Im} \Pi\left(M_{\rho}^{2}\right)$, where $\Gamma_{\rho} \equiv \Gamma_{\rho}\left(M_{\rho}^{2}\right)$, and from Eq.(12) there follows

$$
\Gamma_{\rho}=\frac{g_{\rho \pi \pi}^{2}}{48 \pi} \frac{1}{M_{\rho}^{2}}\left(M_{\rho}^{2}-4 \mu_{\pi}^{2}\right)^{\frac{3}{2}}
$$


which is the standard kinematical relation between width and coupling of a vector and two pseudoscalar particles [10]. Notice that this results follows automatically in the KLZ model, i.e. it has not been imposed as a constraint. Near the rho-meson peak, where $\Pi(s)$ is largely purely imaginary, the s-dependent width which follows from Eqs. (12) and (15) is

$$
\left.\Gamma_{\rho}(s)\right|_{K L Z}=\frac{M_{\rho} \Gamma_{\rho}}{\sqrt{s}}\left[\frac{s-4 \mu_{\pi}^{2}}{M_{\rho}^{2}-4 \mu_{\pi}^{2}}\right]^{\frac{3}{2}},
$$

which is precisely the momentum dependent Gounaris-Sakurai width [10]. This is known to provide an excellent fit to the data in this region [6].

Our results are shown in Fig.2 (solid line) together with the experimental data [11] and the prediction from tree-level VMD (dotted curve). The latter provides a poor fit to the data as evidenced from the resulting chi-square per degrees of freedom $\chi_{F}^{2}=5.0$, while Eq.(11) gives the optimal value $\chi_{F}^{2}=1.1$. In addition, the mean-square radius of the pion obtained from Eq.(35) is $\left\langle r_{\pi}^{2}>=0.46 \mathrm{fm}^{2}\right.$, to be compared with a much smaller result from tree-level $\operatorname{VMD}<r_{\pi}^{2}>=6 / M_{\rho}^{2}=0.39 \mathrm{fm}^{2}$, and the experimental value $<r_{\pi}^{2}>=0.439 \pm 0.008 \mathrm{fm}^{2}$. In summary, the KLZ one-loop level contributions to the pion form factor turn out to be reasonable corrections to the leading order result. This is in spite of KLZ being a strong interaction theory. This is due to the relatively mild coupling $\left(g_{\rho \pi \pi} \simeq 5\right)$, together with a large loop suppression factor $\left((1 / 4 \pi)^{2}\right)$, as seen from Eq. (6). Increasing powers of this suppression factor are expected at higher orders in perturbation theory.

In the mean time, the KLZ model has been used to calculate the scalar radius of the pion at next to leading order (one loop) in perturbation theory. See [12] for details. The result provides additional support for the KLZ theory as a viable platform to compute corrections to VMD systematically in perturbation theory.

The pion scalar form factor $\Gamma_{\pi}\left(q^{2}\right)$ is given in terms of the pion matrix elements of the scalar operator $J_{S}=m_{u} \bar{u} u+m_{d} \bar{d} d$ according to

$$
\Gamma_{\pi}\left(q^{2}\right)=\left\langle\pi\left(p_{2}\right)\left|J_{S}\right| \pi\left(p_{1}\right)\right\rangle
$$

where $q^{2}=\left(p_{2}-p_{1}\right)^{2}$. The associated quadratic scalar radius

$$
\Gamma_{\pi}\left(q^{2}\right)=\Gamma_{\pi}(0)\left[1+\frac{1}{6}\left\langle r_{\pi}^{2}\right\rangle_{S} q^{2}+\cdots\right]
$$




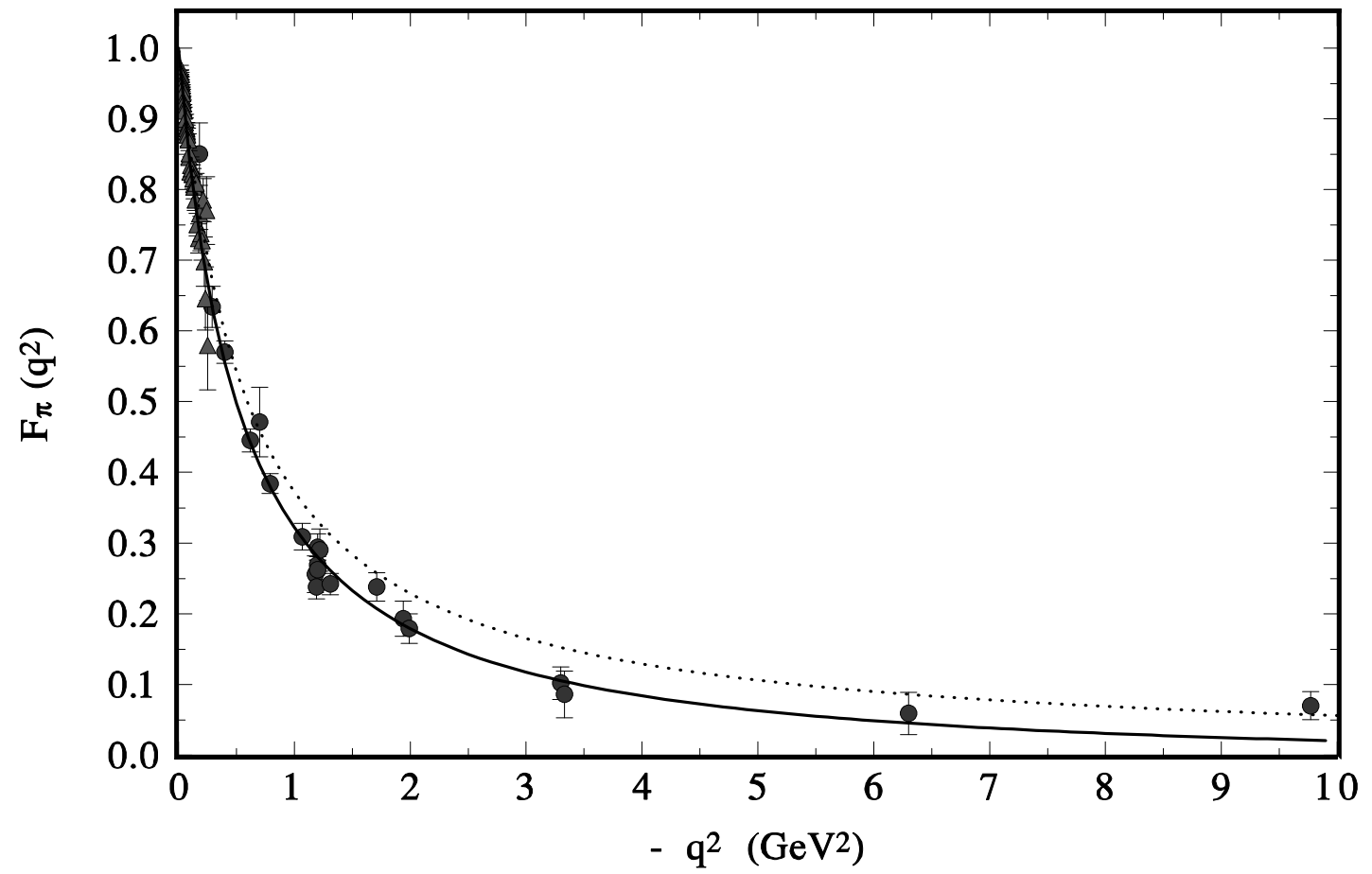

FIG. 2: Pion form factor data together with the KLZ prediction, Eq.(11) (solid line), and the tree-level VMD result (dotted line).

is important in chiral perturbation theory since it fixes $\bar{l}_{4}[13]$.

Our one loop corrections in the KLZ model give

$$
\left\langle r_{\pi}^{2}\right\rangle_{S}=0.4 \mathrm{fm}^{2} ; \bar{l}_{4}=3.4
$$

which are close to the current values [14] $\left\langle r_{\pi}^{2}\right\rangle_{s} \simeq 0.5-0.7 \mathrm{fm}^{2}$ and $\bar{l}_{4} \simeq 4.0-5.1$. Higher loop corrections $(N N L O)$ are currently been analized, since they are the main uncertainty in this determination.

Acknowledgements: We acknowledge support by Fondecyt under grants 1051067, 
7070178, and by Centro de Estudios Subatómicos (Chile) and by NRF (South Africa)

[1] N.M. Kroll, T.D. Lee, B. Zumino, Phys. Rev. 175 (1967) 1376; J.H. Lowenstein, B. Schroer,Phys. Rev. D 6 (1972) 1553.

[2] J.J. Sakurai, Ann. Phys. (N.Y.) 11 (1960) 1; ibid. Currents and Mesons, University of Chicago Press (1969).

[3] H. van Hees, hep-th/0305076 (unpublished); H. Ruegg, M. Ruiz-Altaba, Int. J. Mod. Phys. A 19 (2004) 3265.

[4] C. Gale, J. Kapusta, Nucl. Phys. B 357 (1991) 65.

[5] G. Gounaris, J.J. Sakurai, Phys. Rev. Lett. 21 (1968) 244; see also M. Gourdin, Phys. Rep. $11 \mathrm{C}(1974) 29$.

[6] M. Davier, A. Höcker, Z. Zhang, Rev. Mod. Phys. 78 (2006) 1043.

[7] C. A. Dominguez, J. I. Jottar, M. Loewe, and B. Willers, Phys. Rev. D 76 (2007) 095002.

[8] C. Quigg, Gauge Theories of Strong, Weak, and Electromagnetic Interactions, Benjamin (1983).

[9] Particle Data Group, J. Phys. G 33 (2006) 1.

[10] H.M. Pilkuhn, Relativistic Particle Physics, Springer Verlag (1979).

[11] C.J. Bebek et al., Phys. Rev. D 17 (1978) 1693; S.R. Amendolia et al., Nucl. Phys. B 277 (1986) 168;, J. Volmer et al., Phys. Rev. Lett. 86 (2001) 1713.

[12] C. A. Dominguez, M. Loewe, and B. Willers, hep-ph 0808.0823, to appear in Phys. Rev. D.

[13] S.Scherer, Introduction to Chiral Perturbation Theory, Adv. Nucl. Phys. 27 (2003) 277; J. Gasser, Lect. Notes Phys. 629 (2004) 1.

[14] J. A. Oller and L. Roca, Phys. lett. B 651 (2007) 139. 